\title{
Fisher entropy, Rényi entropy power and quantum phase transition in the Dicke model
}

\author{
Á. Nagy \\ Department of Theoretical Physics, University of Debrecen, H-4010 Debrecen, Hungary \\ E. Romera \\ Departamento de Física Atómica, Molecular y Nuclear and \\ Instituto Carlos I de Física Teórica y Computacional, \\ Universidad de Granada, Fuentenueva s/n, 18071 Granada, Spain
}

(Dated: May 25, 2011)

\begin{abstract}
Fisher entropy, Rényi entropy power and Fisher-Rényi entropy product are presented for the Dicke model. There is a quantum phase transition in this quantum optical model. It is pointed out that there is an abrupt change in the Fisher entropy, Rényi entropy power, the Fisher, Shannon and Rényi lengths at the transition point. It is found that these quantities diverge as the characteristic length: $\left|\lambda_{c}-\lambda\right|^{-1 / 4}$ around the critical value of the coupling strength $\lambda_{c}$ for any value of the parameter $\beta$.
\end{abstract}


Information theoretical concepts have turned to be extremely useful in studying physical systems. Especially Rényi and Fisher information are of current interest. Rényi entropy has been applied in several fields, e. g. quantum entanglement [1], quantum communications protocols [2], atomic ionization properties [3], quantum revivals [4] or localization properties [5]. Fisher information [6] has been attracting considerable interest in a variety of scientific subjects, such as, neuroscience [7], density functional theory [8-10], or atoms and molecules [12-26]. Several useful entropic uncertainty relations have been presented [27-33].

Consider a D-dimensional distribution function $f(\mathbf{r})$, with $f(\mathbf{r})$ nonnegative and $\int f(\mathbf{r}) d \mathbf{r}=$ 1 , where $\mathbf{r}$ stands for $r_{1}, \ldots, r_{D}$. The Fisher information of the probability density function $f$ is given by

$$
I_{f} \equiv \int \frac{|\nabla f(\mathbf{r})|^{2}}{f(\mathbf{r})} d \mathbf{r}
$$

The Rényi entropy of order $\beta$ is defined by

$$
R_{f}^{\beta} \equiv \frac{1}{1-\beta} \ln \int f^{\beta}(\mathbf{r}) d \mathbf{r} \quad \text { for } \quad 0<\beta<\infty \quad \beta \neq 1 .
$$

It is straightforward to obtain the Rényi entropy limit when $\beta \rightarrow 1$ which gives the Shannon entropy

$$
S_{f} \equiv-\int f(\mathbf{r}) \ln f(\mathbf{r}) d \mathbf{r} .
$$

The Rényi entropy power of index $\beta$ is defined by

$$
\begin{aligned}
N_{f}^{\beta} \equiv & \left(\frac{\beta}{2 \beta-1}\right)^{\frac{2 \beta-1}{\beta-1}} \frac{1}{2 \pi} \exp \left(\frac{2}{D} R_{f}^{\beta}\right) \\
& =\alpha^{1 /(1-\alpha)} \frac{1}{2 \pi} \exp \left(\frac{2}{D} R_{f}^{\beta}\right)
\end{aligned}
$$

whith $\alpha^{-1}+\beta^{-1}=2$ as an extension of Shannon entropy power

$$
N_{f} \equiv \frac{1}{2 \pi e} e^{\frac{2}{D} S_{f}}
$$

If $\beta$ tends to 1 the Rényi entropy power results the Shannon entropy power.

The Fisher-Rényi information product is defined by

$$
P_{f}^{\beta}=\frac{1}{D} N_{f}^{\beta} I_{f} \quad \text { with } \quad \beta \in(1 / 2,1]
$$

as an extension of the Fisher-Shannon product [20]. 
The spreading measure of a probability density is generally given by the simple root-mean square or standard deviation

$$
\Delta x=\left(\left\langle x^{2}\right\rangle-\langle x\rangle^{2}\right)^{1 / 2}
$$

In fact, probability density can be characterized by information-theoretic-based spreading measures. The definition of Fisher, Shannon and Rényi lengths are

$$
\begin{aligned}
& l_{I}=\frac{1}{\sqrt{I}} \\
& l_{S}=e^{2 S / D}
\end{aligned}
$$

and

$$
l_{R^{\beta}}=e^{2 R^{\beta} / D},
$$

respectively.

The Dicke model is a quantum optical model that describes a single-mode bosonic field interacting with an ensemble of $N$ two-level atoms. There is a quantum phase transition (QPT) in the $N \rightarrow \infty$ limit. The Dicke model has an analytical solution presented by Emary and Brandes $[34,35]$. The single-mode Dicke Hamiltonian has the form

$$
H=\omega_{0} J_{z}+\omega a^{+} a+\frac{\lambda}{\sqrt{2 j}}\left(a^{+}+a\right)\left(J_{+}+J_{-}\right)
$$

where $J_{z}, J_{ \pm}$are the angular momentum operators for a pseudospin of length $j=N / 2$ (to describe the ensemble of two-level atoms of level-splitting $\left.\omega_{0}\right)$ and $a$ and $a^{+}$are the bosonic operators of the field (the bosonic mode has a frequency $\omega$ ).

In the thermodynamic limit, where the number of atoms become infinite $(N, j \rightarrow \infty)$, there is a QPT at a critical value of the atom-field coupling strength $\lambda_{c}=\frac{1}{2} \sqrt{\omega \omega_{0}}$. There are two phases: normal phase $\left(\lambda<\lambda_{c}\right)$ and superradiant phase $\left(\lambda>\lambda_{c}\right)$.

Using the Holstein-Primakoff representation $[36,37]$ the Hamiltonian can be diagonalized. In the normal phase it has the form

$$
H^{(1)}=\frac{1}{2}\left(\varepsilon_{-}^{(1) 2} q_{1}^{2}+p_{1}^{2}+\varepsilon_{+}^{(1) 2} y^{2}+p_{2}^{2}-\omega_{0}-\omega\right)-j \omega_{0}
$$

The energies of oscillator modes are

$$
\varepsilon_{ \pm}^{(1) 2}=\frac{1}{2}\left[\omega^{2}+\omega_{0}^{2} \pm \sqrt{\left(\omega_{0}^{2}-\omega^{2}\right)^{2}+16 \lambda^{2} \omega \omega_{0}}\right]
$$


As $\varepsilon_{-}^{(1)}$ is real only if $\omega^{2}+\omega_{0}^{2} \geq \sqrt{\left(\omega_{0}^{2}-\omega^{2}\right)^{2}+16 \lambda^{2} \omega \omega_{0}}$ (that corresponds to $\lambda \leq \lambda_{c}$ ), the normal phase with Hamiltonian $H^{(1)}$ exists if $\lambda \leq \lambda_{c}$. In the super-radiant phase the Holstein-Primakoff transformation [34] leads to the final diagonal form

$$
H^{(2)}=\varepsilon_{-}^{(2)} e_{1}^{+} e_{1}+\varepsilon_{+}^{(2)} e_{2}^{+} e_{2}-j\left(\frac{2 \lambda^{2}}{\omega}+\frac{\omega_{0}^{2} \omega}{8 \lambda^{2}}\right)+\frac{1}{2}\left(\varepsilon_{+}^{(2)}+\varepsilon_{-}^{(2)}-\frac{\omega_{0}}{2 \mu}(1+\mu)-\omega-\frac{2 \lambda^{2}}{\omega}(1-\mu)\right),
$$

where

$$
\mu=\frac{\lambda_{c}^{2}}{\lambda^{2}}
$$

$e_{1}$ and $e_{2}$ are the new Bose operators corresponding to the new rotated, decoupled oscillators (see details in [34]). The oscillator energies are

$$
\varepsilon_{ \pm}^{(2) 2}=\frac{1}{2}\left[\omega^{2}+\frac{\omega_{0}^{2}}{\mu^{2}} \pm \sqrt{\left(\frac{\omega_{0}^{2}}{\mu^{2}}-\omega^{2}\right)^{2}+4 \omega^{2} \omega_{0}^{2}}\right]
$$

The excitation energy $\varepsilon_{-}^{(2)}$ is real if $\lambda \geq \lambda_{c}$. In the super-radiant phase the spectrum is doubly degenerate, the symmetry changes at the critical point $\lambda_{c}$.

The wave function can be analytically given in the Dicke model [34, 35]. In the normal phase it has the form

$$
\Psi^{(1)}\left(q_{1}, q_{2}\right)=G_{-}^{(1)}\left(q_{1}\right) G_{+}^{(1)}\left(q_{2}\right)
$$

where

$$
G_{ \pm}^{(1,2)}(q)=\left(\frac{\varepsilon_{ \pm}^{(1,2)}}{\pi}\right)^{1 / 4} \exp \left[-\frac{\varepsilon_{ \pm}^{(1,2)}}{2} q^{2}\right]
$$

are normalized Gaussian functions. In the original x-y representation the wave function can be obtained with a rotation of the coordinate system

$$
x=q_{1} \cos \gamma^{(1)}+q_{2} \sin \gamma^{(1)}, \quad y=-q_{1} \sin \gamma^{(1)}+q_{2} \cos \gamma^{(1)}
$$

where the angle $\gamma^{(1)}$ is defined as

$$
\tan \left(2 \gamma^{(1)}\right)=\frac{4 \lambda \sqrt{\omega \omega_{0}}}{\omega_{0}^{2}-\omega^{2}}
$$

In the super-radiant phase the wave function is given by

$$
\Psi^{(2)}\left(Q_{1}, Q_{2}\right)=G_{-}^{(2)}\left(Q_{1}\right) G_{+}^{(2)}\left(Q_{2}\right)
$$


In the original $x-y$ representation

$$
\begin{aligned}
& Q_{1}=(x-\sqrt{2 \tilde{\alpha} / \omega}) \cos \gamma^{(2)}-\sqrt{\omega_{0} / \widetilde{\omega}}\left(y+\sqrt{2 \tilde{\beta} / \omega_{0}}\right) \sin \gamma^{(2)}, \\
& Q_{2}=(x-\sqrt{2 \tilde{\alpha} / \omega}) \sin \gamma^{(2)}+\sqrt{\omega_{0} / \widetilde{\omega}}\left(y+\sqrt{2 \tilde{\beta} / \omega_{0}}\right) \cos \gamma^{(2)},
\end{aligned}
$$

where

$$
\begin{aligned}
\tan \left(2 \gamma^{(2)}\right) & =2 \omega \omega_{0} \mu^{2} /\left(\omega_{0}^{2}-\mu^{2} \omega^{2}\right), \\
\widetilde{\omega} & =(1+\mu) \omega_{0} / 2 \mu .
\end{aligned}
$$

and

$$
\sqrt{\tilde{\alpha}}=\frac{2 \lambda}{\omega} \sqrt{\frac{j}{2}\left(1-\mu^{2}\right)} \quad \sqrt{\tilde{\beta}}=\sqrt{j(1-\mu)} .
$$

From the wave function we can immediatelly obtain the probability density as $|\Psi|^{2}$. Then substituting the probability density into Eq. (1) the Fisher information has the form

$$
I^{(i)}=2\left(\varepsilon_{-}^{(i)}+\varepsilon_{+}^{(i)}\right)
$$

where $i=1$ in the normal and $i=2$ in the super-radiant phase. The Fisher length reads as

$$
l_{I}^{(i)}=\frac{1}{\sqrt{2\left(\varepsilon_{-}^{(i)}+\varepsilon_{+}^{(i)}\right)}},
$$

The Rényi entropy has recently been calculated [38]:

$$
R^{(i) \beta}=\ln \pi-\ln \sqrt{\varepsilon_{+}^{(i)} \varepsilon_{-}^{(i)}}-\frac{\ln \beta}{1-\beta} .
$$

The limit $\beta \rightarrow 1$ gives the Shannon entropy:

$$
R^{(i) \beta}=\ln \pi-\ln \sqrt{\varepsilon_{+}^{(i)} \varepsilon_{-}^{(i)}}+1 .
$$

The Rényi length of order $\beta$ reads

$$
l_{R^{(i) \beta}}=\frac{\pi}{\sqrt{\varepsilon_{+}^{(i)} \varepsilon_{-}^{(i)}}} \beta^{-\frac{1}{11-\beta}} .
$$

The limit $\beta \rightarrow 1$ gives the Shannon length

$$
l_{S}^{(i)}=\frac{\pi}{\sqrt{\varepsilon_{+}^{(i)} \varepsilon_{-}^{(i)}}} e .
$$




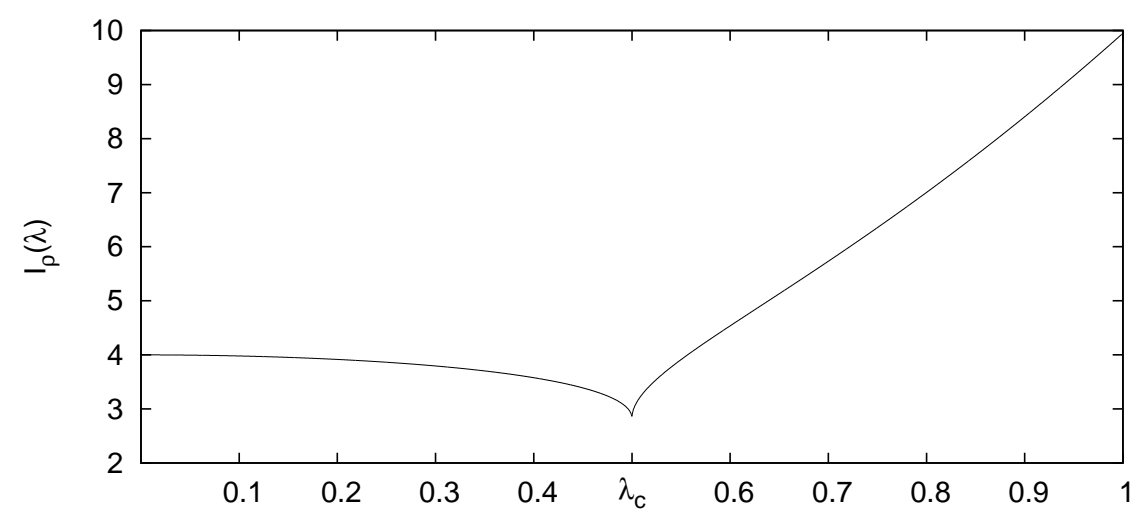

FIG. 1. Fisher information of the ground state of the Dicke Hamiltonian in the thermodynamic limit as a function of the coupling $\lambda$ for $\omega=\omega_{0}=1$.

Substitution of the Rényi entropy (29) into (4) leads to the Rényi entropy power

$$
N^{(i) \beta}=\frac{\eta}{2} \frac{1}{\sqrt{\varepsilon_{-}^{(i)} \varepsilon_{+}^{(i)}}},
$$

where

$$
\eta=\left(\frac{\beta}{2 \beta-1}\right)^{\frac{2 \beta-1}{\beta-1}} \beta^{-\frac{1}{11-\beta}}
$$

From Eqs. (6) and (29) the Fisher-Rényi information product takes the form

$$
P^{(i) \beta}=\frac{\eta}{2} \frac{\varepsilon_{-}^{(i)}+\varepsilon_{+}^{(i)}}{\sqrt{\varepsilon_{-}^{(i)} \varepsilon_{+}^{(i)}}}
$$

and its characteristic length is given by

$$
l_{P^{\beta}}=P^{\beta}
$$

Figs 1-3 present the Fisher entropy, the Rényi entropy power and the Fisher-Rényi information product of order 2. These quantities exhibit an abrupt change at the critical point. The behaviour is similar at any value of $\beta$ that can be explained as follows. The characteristic length scale of the system is defined by

$$
l_{-}=\frac{1}{\sqrt{\varepsilon_{-}}} .
$$

In the vicinity of the critical point $\varepsilon_{-} \varepsilon_{+}$take the form [34]

$$
\varepsilon_{-}\left(\lambda \rightarrow \lambda_{c}\right) \sim \sqrt{\frac{32 \lambda_{c}^{3} \omega^{2}}{16 \lambda_{c}^{4}+\omega^{4}}} \quad\left|\lambda_{c}-\lambda\right|^{1 / 2}, \quad \varepsilon_{+}\left(\lambda \rightarrow \lambda_{c}\right) \sim \sqrt{\omega_{0}^{2}+\omega^{2}} .
$$




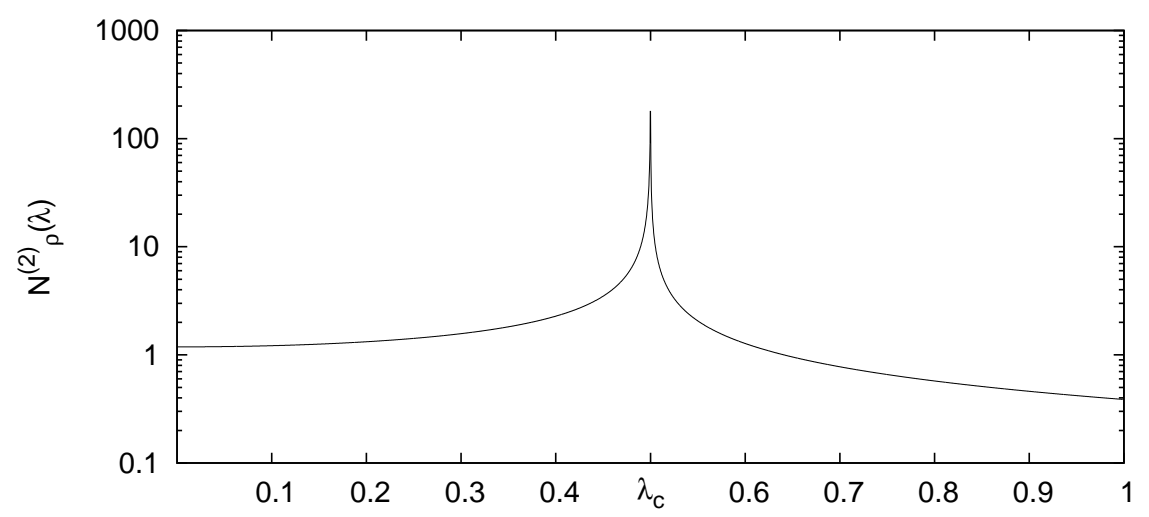

FIG. 2. Rényi entropy power of order 2 (characteristic lenght) of the ground state of the Dicke Hamiltonian in the thermodynamic limit as a function of the coupling $\lambda$ for $\omega=\omega_{0}=1$.

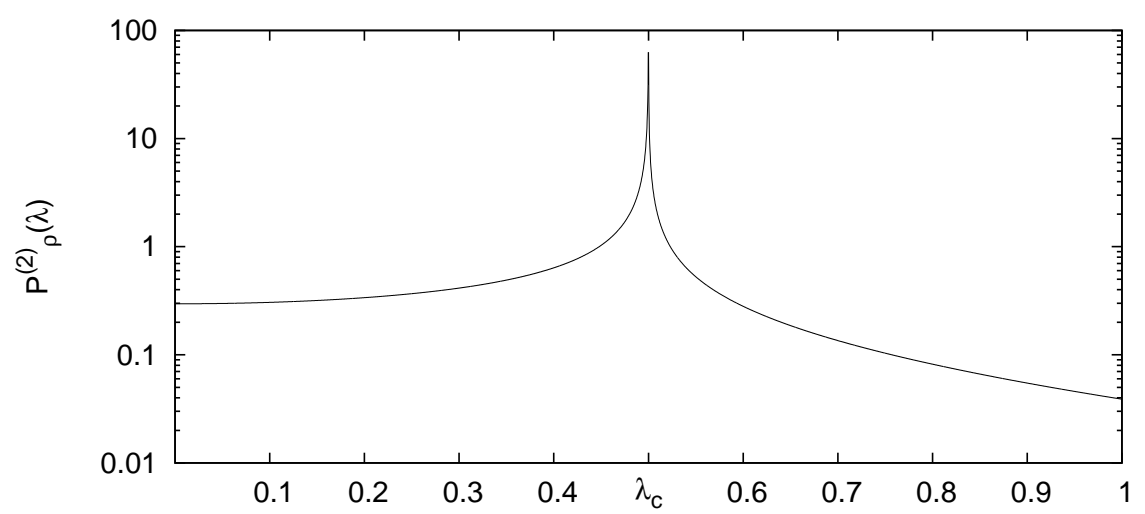

FIG. 3. Fisher-Rényi information product of order 2 (characteristic lenght) of the ground state of the Dicke Hamiltonian in the thermodynamic limit as a function of the coupling $\lambda$ for $\omega=\omega_{0}=1$.

Therefore

$$
\ln l_{-} \propto-\ln \left|\lambda_{c}-\lambda\right|
$$

as $\lambda \rightarrow \lambda_{c}$. From Eqs. (35),(30) and (31) we obtain that

$$
l_{P^{(\beta)}} \propto l_{R^{\beta}} \propto l_{S} \propto l_{-} \propto\left|\lambda_{c}-\lambda\right|^{-1 / 4} .
$$

That is, the Fisher-Rényi product, Rényi and Shannon lengths are propotional to the characteristic length $l_{-}$and diverge as $\left|\lambda_{c}-\lambda\right|^{-1 / 4}$ around the critical point. Eqs. (32) and (37) leads to

$$
N^{\beta} \propto l_{-} \propto\left|\lambda_{c}-\lambda\right|^{-1 / 4}
$$

In figure 4 we can see the Fisher characteristic length that has a relative maximum in the critical point. 


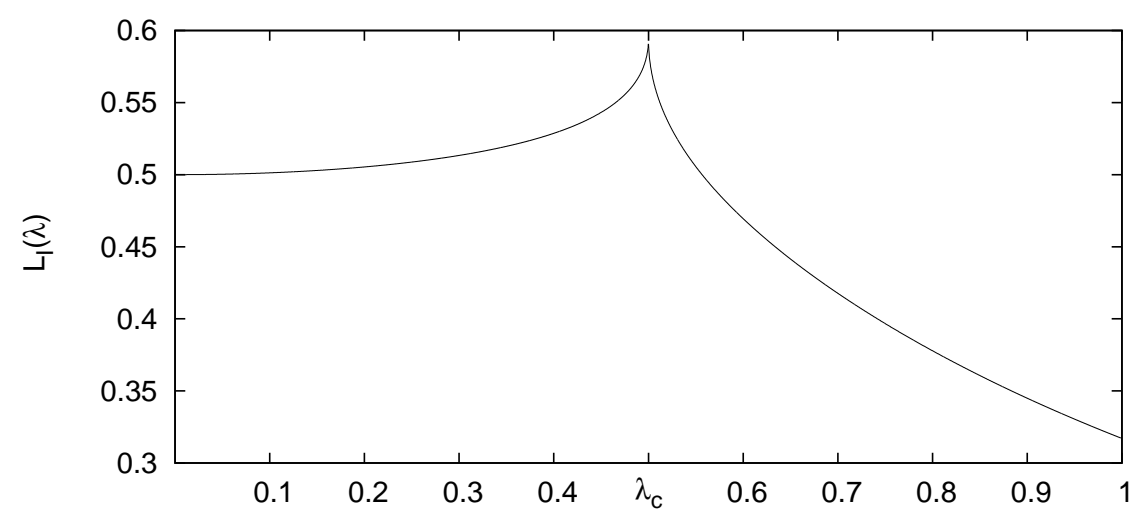

FIG. 4. Fisher characteristic lenght of the ground state of the Dicke Hamiltonian in the thermodynamic limit as a function of the coupling $\lambda$ for $\omega=\omega_{0}=1$.

In summary, we have shown that there is an abrupt change in the Fisher-Rényi information product, Rényi entropy power, Shannon and Rényi lengths at the transition point of the Dicke model. It is found that these quantities diverge as the characteristic length: $\left|\lambda_{c}-\lambda\right|^{-1 / 4}$ around the critical value of the coupling strength $\lambda_{c}$. In the Rényi lengths and the Rényi entropy power there is no dependence on the value of the parameter $\beta$ in the vicinity of the critical point. For the Fisher lenght we hava a maximum as an indicator of the quantum phase transition.

\section{ACKNOWLEDGEMENTS}

The work is supported by the TAMOP 4.2.1/B-09/1/KONV-2010-0007 project. The project is co-financed by the European Union and the European Social Fund. Grant OTKA No. K 67923 is also gratefully acknowledged.

[1] O. Gühne and M. Lewenstein, Phys. Rev. A 70 (2004) 022316; G. Adesso, A. Serafini and F. Illuminati, Phys. Rev. A 70 (2004) 022318; A. Bovino, G. Castagnolli, A. Ekert, P. Horodecki, C. M. Alves and A. V. Serfienko, Phys. Rev. Lett. 95 (2005) 240407.

[2] R. Renner, N. Gisin and B. Kraus, Phys. Rev. A 72 (2005) 012332; V. Giovanetti and S. Lloyd, Phys. Rev. A 69 (2004) 062307.

[3] E. Romera and A. Nagy Phys. Lett. A, in press (2008)

[4] E. Romera and F. de los Santos, Phys. Rev. A, in press (2008). 
[5] D. G. Arbo, C. O. Reinhold, J. Burgdörfer, A. K. Pattanayak, C. L. Stokely, W. Zhao, J. C. Lancaster and F. B. Dunning, Phys. Rev. A 67 (2003) 063401.

[6] R. A. Fisher, Proc. Cambridge Philos. Soc.22 (1925) 700.

[7] S. A. Solla, Plos Biology, 4 (2006) e122.

[8] S. B. Sears, R. G. Parr, and U. Dinur, Isr. J. Chem. 19 (1980) 165.

[9] R. Nalewajski, Chem. Phys. Lett. 372 (2003) 28.

[10] A. Nagy, J. Chem. Phys. 119 (2003) 9407.

[11] B. R. Frieden, Physics form Fisher Information. A Unification (Cambridge University Press, Cambridge, 1998).

[12] B. R. Frieden, Am. J. Phys. 57 (1989) 1004.

[13] M. Reginatto, Phys. Rev. A 58 (1998) 1775.

[14] R. Nalewajski, Chem. Phys. Lett. 372 (2003) 28.

[15] Á. Nagy, J. Chem. Phys. 119 (2003) 9401.

[16] E. Romera, P. Sánchez-Moreno and J. S. Dehesa, Chem. Phys. Lett. 414 (2005) 468.

[17] Á. Nagy, Chem. Phys. Lett.425 (2006) 157.

[18] Á. Nagy and K. D. Sen, Phys. Lett. A 360 (2006) 291.

[19] I. Hornyák and Á. Nagy, Chem. Phys. Lett. 437 (2007) 132.

[20] E. Romera and J. S. Dehesa, J. Chem. Phys. 120 (2004) 8906.

[21] E. Romera, Mol. Phys. 100 (2002) 3325.

[22] Á. Nagy and K. D. Sen, Phys. Lett. A 360 (2006) 291.

[23] S. B. Liu, J. Chem. Phys. 126 (2007) 191107.

[24] Á. Nagy, Chem. Phys. Lett. 449 (2007) 212.

[25] Á. Nagy and S. B. Liu, Phys. Lett. A 372 (2008) 1654.

[26] J. B. Szabó, K. D. Sen and Á. Nagy, Phys. Lett. A 372 (2008) 2428.

[27] I. Bialynicki-Birula, Phys. Rev. A 74, 052101 (2006).

[28] I. Bianilicki-Birula and J. Mycielski, Commun. Math. Phys. 44, 129 (1975); W. Beckner, Ann. Math. 102, 159 (1975).

[29] S. R. Gadre, S. B. Sears,S. J. Chakravorty and R. D. Bendale, Phys. Rev. A 32, 2602 (1985).

[30] A. Dembo, T. M. Cover, and J. A. Thomas, IEEE Trans. Inf. Theory 37, 1501 (1991).

[31] C. Vignat, J. F. Bercher, Phys. Lett. A 312 (2003) 27.

[32] S. Zozor, M. Portesi, and C. Vignat arXiv 07093011v1 (2007). 
[33] E. Romera and Á. Nagy, Phys. Lett. A 372, 6823 (2008).

[34] C. Emary and T. Brandes, Phys. Rev. E 67, 066203 (2003).

[35] C. Emary and T. Brandes, Phys. Rev. Lett. 90, 044101 (2003).

[36] T. Holstein and H. Primakoff, Phys. Rev. 58, 1098 (1949).

[37] E. Ressayre and A. Tallet, Phys. Rev. A 11, 981 (1975).

[38] E. Romera and Á. Nagy, submitted 